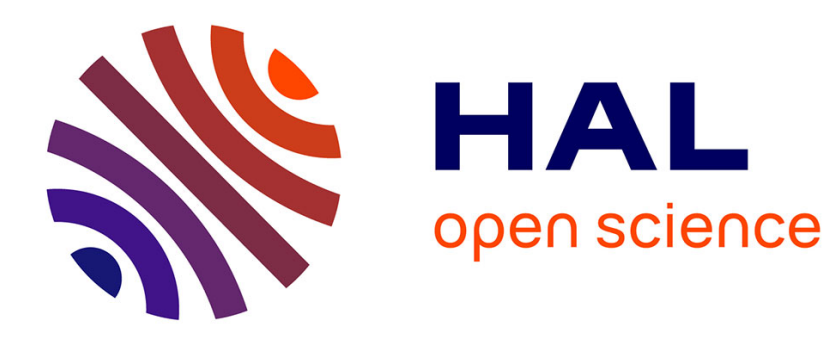

\title{
Update of the hadronic contribution to the QED vacuum polarization
}

\author{
Hans Burkhardt, B. Pietrzyk
}

\section{To cite this version:}

Hans Burkhardt, B. Pietrzyk. Update of the hadronic contribution to the QED vacuum polarization. Physics Letters B, 2001, 513, pp.46-52. in2p3-00009886

\section{HAL Id: in2p3-00009886 https://hal.in2p3.fr/in2p3-00009886}

Submitted on 2 Aug 2001

HAL is a multi-disciplinary open access archive for the deposit and dissemination of scientific research documents, whether they are published or not. The documents may come from teaching and research institutions in France or abroad, or from public or private research centers.
L'archive ouverte pluridisciplinaire HAL, est destinée au dépôt et à la diffusion de documents scientifiques de niveau recherche, publiés ou non, émanant des établissements d'enseignement et de recherche français ou étrangers, des laboratoires publics ou privés. 


\title{
Update of the hadronic contribution to the QED vacuum polarization
}

\author{
H. Burkhardt ${ }^{\mathrm{a}}$, B. Pietrzyk ${ }^{\mathrm{b}}$

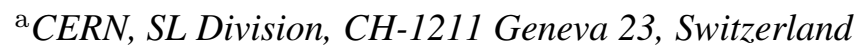 \\ ${ }^{\mathrm{b}}$ Laboratoire de Physique des Particules LAPP, IN2P3-CNRS, F-74941 Annecy-le-Vieux \\ Cedex, France
}

\begin{abstract}
We have updated the evaluation of the hadronic contribution to the running of the QED fine structure constant. It is obtained from a dispersion integral over a parametrization of the measured cross section of $\mathrm{e}^{+} \mathrm{e}^{-} \rightarrow$ hadrons. We find this contribution to be $\Delta \alpha_{\text {had }}^{(5)}(s)$ $=0.02761 \pm 0.00036$ at $\mathrm{s}=m_{Z}^{2}$ corresponding to $1 / \alpha^{(5)}\left(m_{Z}^{2}\right)=128.936 \pm 0.046$. The improved experimental accuracy is mainly due to recent BES measurements. We have also updated and parametrized the hadronic contribution to $\alpha$ as a function of energy.
\end{abstract}

Email addresses: Helmut.Burkhardt@cern.ch(H. Burkhardt), pietrzyk@lapp.in2p3.fr(B. Pietrzyk).

URLs: http://home.cern. ch/hbu/aqed/aqed.html (H. Burkhardt), http: //alephwww. cern. ch/ pietrzyk/ (B. Pietrzyk). 


\section{Introduction}

Considerable progress has been made in understanding of the electroweak gauge theory of elementary particle interactions in the last decade. Precision high energy data from LEP, SLC and Tevatron have clearly established the existence of electroweak radiative corrections. The dominant correction is provided by the running of the electromagnetic coupling constant $\alpha$ from its value at vanishing momentum transfer to high energies.

A combined analysis of the high energy experimental data is performed by the LEP electroweak working group [1]. Recent results from global fits have been reported in [2]. The results are perfectly consistent with the standard model and allow a determination of the standard model Higgs boson mass from radiative corrections. The Higgs mass obtained in these fits depends mainly on the value of the top mass and the hadronic contribution of the five lighter quarks $\Delta \alpha_{\text {had }}^{(5)}(s)$ to the running of the QED fine structure constant.

The running of $\alpha$ can be expressed as

$$
\alpha(s)=\frac{\alpha(0)}{1-\Delta \alpha_{l}(s)-\Delta \alpha_{\text {had }}^{(5)}(s)-\Delta \alpha_{\text {top }}(s)} .
$$

The leptonic contribution $\Delta \alpha_{l}(s)$ is known with high accuracy [3], so that its uncertainty can be neglected in the present analysis. The contribution from the top quark loops $\Delta \alpha_{\text {top }}(s)$ is treated separately, such that the top quark mass can be treated as parameter in standard model fits.

The main subject of this paper is the up-to-date estimate of the contribution from the lighter five quark flavours $\Delta \alpha_{\text {had }}^{(5)}(s)$. It is obtained as dispersion integral [4] (Kramers-Kronig rule; the $P$ stands for the principal value of the integral )

$$
\Delta \alpha_{\text {had }}(s)=-\frac{\alpha s}{3 \pi} P \int_{4 m_{\pi}^{2}}^{\infty} \frac{R_{\mathrm{had}}\left(s^{\prime}\right)}{s^{\prime}\left(s^{\prime}-s\right)} d s^{\prime} .
$$

$R_{\text {had }}$ is the measured QED cross-section of the process $\mathrm{e}^{+} \mathrm{e}^{-} \rightarrow$ hadrons, normalized to the QED cross-section for lepton-pair production. The integration can be done numerically or even analytically for energy intervals in which $R_{\text {had }}(s)$ is parametrized by straight lines. The uncertainty in the result obtained from Eq. (1) is almost entirely due to experimental errors in the determination of $R_{\text {had }}(s)$, and in particular free of assumptions on light quark masses and nonperturbative QCD.

Computational uncertainties, introduced by the particular choice of the parametrization and algorithm used, are negligible compared to the experimental uncertainty in 
the data. This has been checked by comparing different programs and parametrization methods [5].

As in our previous analysis published in 1995 [6], we prefer to represent $R_{\text {had }}$ by simple parametrizations, like broad averages and straight lines in the continuum and rely, whenever available, on published world averages. The uncertainty in our previous analysis was mainly due to the poor knowledge of the cross sections for c.m.s. energies $(\sqrt{s})$ from 1 to $5 \mathrm{GeV}$ (see Fig. 2 in [6] ). Recently, the BES Collaboration [7,8] measured $R_{\text {had }}(s)$ between 2 and $5 \mathrm{GeV}$ with substantially improved precision compared to previous measurements.

\section{Updated analysis}

We updated $R_{\text {had }}(s)$ and recalculated $\Delta \alpha(s)$ using all currently available data. Our parametrization of $R_{\text {had }}$ including resonances for centre-of-mass energies up to 10 $\mathrm{GeV}$ is shown as a solid line in Fig. 1. The relative error of the continuum contribution of $R_{\text {had }}$ is shown as a band and is given in numbers at the bottom of the figure. Measurements of various experiments are shown with their statistical errors.

The contributions from the $\rho$, narrow resonances and different energy regions to $\Delta \alpha_{\text {had }}^{(5)}\left(m_{Z}^{2}\right)$ are summarized in Table 1 and illustrated in Fig. 2. Even with the recent significant improvement in measurements at lower energies, it is still the 1 to $5 \mathrm{GeV}$ energy range which dominates the uncertainty in $\Delta \alpha\left(m_{Z}^{2}\right)$.

Table 1

\begin{tabular}{|c|c|c|c|}
\hline $\begin{array}{c}\text { Range } \sqrt{s}, \\
\quad \mathrm{GeV}\end{array}$ & $\Delta \alpha$ & $\begin{array}{c}\text { Relative } \\
\text { error }\end{array}$ & Comments \\
\hline$\rho$ & 0.00350 & $2.3 \%$ & including CMD-2 at VEPP-2M \\
\hline Narrow resonances & 0.00184 & $3.1 \%$ & PDG, undressed width, without $\psi$ ". \\
\hline $1.05-2.0$ & 0.00156 & $1.5 \%$ & as in 1995 paper \\
\hline $2.0-5.0$ & 0.00381 & $5.9 \%$ & including BES, with $\psi " .$. \\
\hline $5-7$ & 0.00183 & $6 \%$ & as in 1995 paper \\
\hline $7-12$ & 0.00304 & $1.4 \%$ & including CLEO \\
\hline \multirow[t]{2}{*}{$>12$} & 0.01203 & $0.2 \%$ & third-order QCD \\
\hline & 0.02761 & $1.3 \%$ & \\
\hline
\end{tabular}

The changes from the 1995 analysis are given in Table 2 and discussed below:

- In the c.m.s. energy region between 0.61 and $0.96 \mathrm{GeV}$ the results of the pion 
Table 2

Changes in $\Delta \alpha_{\text {had }}^{(5)}\left(m_{Z}^{2}\right)$ from 1995 analysis

\begin{tabular}{lrl|l}
\hline$\Delta \alpha_{\text {had }}^{(5)}\left(m_{Z}^{2}\right)=$ & 0.0280 & \pm 0.0007 & 1995 result \\
\hline & -0.00030 & \\
-0.00008 & & $2-5 \mathrm{GeV}(\mathrm{BES})$ \\
& -0.00001 & & above $12 \mathrm{GeV}(\mathrm{QCD})$ \\
& 0.02761 & \pm 0.00036 & other changes (CMD-2, CLEO, PDG) \\
\hline$\Delta \alpha_{\text {had }}^{(5)}\left(m_{Z}^{2}\right)=$ & &
\end{tabular}

form factor measurements with the CMD-2 detector at the VEPP-2M collider are known to $1.4 \%$ systematic uncertainty. We have used the "hidden local parametrization" of [9], with a small extra bump at $1.2 \mathrm{GeV}$, see Fig. 3. Only statistical errors are shown in the figures. The overall uncertainty of the $\rho$ region integral, including the statistical uncertainty, is $2.3 \%$ (that of $\Gamma_{e e}$ in [9]).

- In the c.m.s. energy region between 2 and $5 \mathrm{GeV}$, results of $R_{\text {had }}$ measurements at 85 different energies have been published recently by the BES Collaboration [7], in addition to the 6 points published earlier [8]. These measurements include the contributions of the charmonium resonances above the $\psi$ '. The number of measured energy points is sufficiently large to allow us to connect and integrate the results directly and to distinguish between correlated systematic uncertainties and uncorrelated statistical errors. For the few overlapping energy points from [7] and [8], we used weighted means. The BES data now allows to obtain the dispersion integral in the 2 to $5 \mathrm{GeV}$ energy range to $5.9 \%$.

This is a substantial improvement, and in fact the most significant single change compared to our 1995 analysis, where $R_{\text {had }}$ in this energy range was only known to $15 \%$. As always in our analysis, the information from the older measurements is kept. Here, this was achieved by a slight rescaling of the BES contribution.

- In the c.m.s. energy region between 7 and $12 \mathrm{GeV}$, a new value for $R_{\text {had }}$ of $3.56 \pm 0.01 \pm 0.07$ was measured by the CLEO collaboration [10] at $\sqrt{s}=10.52$. We use here the mean value of $R_{\text {had }}=3.57$ with the uncertainty of $1.4 \%$ obtained from the combination of the CLEO result with the result of the compilation of other measurements from our 1995 analysis.

- Above the c.m.s. energy of $12 \mathrm{GeV}$, we previously relied on a direct parametrization of PETRA, PEP and TRISTAN data made by D. Haidt [11]. This parametrization was based on second-order QCD with $\alpha_{s}=0.146_{-0.026}^{+0.031}$ at $34 \mathrm{GeV}$ and implied a value of $0.124_{-0.019}^{+0.021}$ at $m_{Z}$. This purely experimental approach was motivated in 1995 by large discrepancies between different values of $\alpha_{s}\left(m_{Z}^{2}\right)$ measured in different experiments [12] ranging from the value 0.108 measured in deep inelastic scattering to the value 0.127 measured from $R_{\text {had }}$ at LEP. A consensus on the value of $\alpha_{s}\left(m_{Z}^{2}\right)$ and on the validity of perturbative QCD emerged since that time. In this paper, we use third-order QCD to describe $R_{\text {had }}$ above 12 $\mathrm{GeV}$, with $\alpha_{s}=0.118 \pm 0.002$ at $m_{Z}$ [13]. This allows a determination of the dispersion integral in this energy range to $0.2 \%$ relative precision.

- Narrow resonances were included as in our 1995 papers [6]. The contribution 
is proportional to the undressed electronic width $\Gamma_{e}$. The values and errors are obtained by slight rescaling of the dressed widths listed in the year 2000 version of the review of particle properties [13]. As mentioned above, the charmonium resonances above the $\psi$ ' are included in this paper as part of the continuum.

\section{Comparison of different estimates in the literature}

Fig. 4 shows a comparison of different estimates of $\Delta \alpha_{\text {had }}^{(5)}\left(m_{Z}^{2}\right)$ since $1989[2,5,6,14-$ 29]. Estimates up to 1995 were discussed in our previous paper [6].

A number of analyses has been made since then, based on more theoretical descriptions of the hadronic cross section. The results are shown as open circles in Fig. 4. Rather compatible results were obtained from 1997 to 1999. It is interesting to see however, that the last of these estimates [29] used results from BES on $R_{\text {had }}(s)$ at low c.m.s. energies and found a significantly lower central value than obtained in the various estimates from 1997 to 1999.

A preliminary value was released by us for the ICHEP 2000 in Osaka [2], in good agreement with the more precise result presented in this paper.

\section{Parametrization as a function of energy}

A simple parametrization of the hadronic contribution to the vacuum polarization $[30,5]$ is used in many computer programs. It is described by the formula

$$
\Delta \alpha_{h}(s) \cong \mathrm{A}+\mathrm{B} \ln (1+\mathrm{C}|s|)
$$

with $s$ or $t$ instead of $s$, both in $\mathrm{GeV}^{2}$. We have updated the parameters $\mathrm{A}, \mathrm{B}$, and $\mathrm{C}$ for five different c.m.s. energy regions. This parametrization is better than 0.2 $\sigma$ in the whole $t$-channel and exact at $m_{Z}=91.1876 \mathrm{GeV}$ in the $s$-channel. The values of the parameters are given in Table 3. Computer code for $\Delta \alpha$ with this parametrization is available from the authors.

\section{Conclusion}

Our updated evaluation of the hadronic contributions to the running of the QED fine structure constant $\alpha$ results in $\Delta \alpha_{\text {had }}^{(5)}\left(m_{Z}^{2}\right)=0.02761 \pm 0.00036$ at s $=m_{Z}^{2}$ or $1 / \alpha^{(5)}\left(m_{Z}^{2}\right)=\left(1-\Delta \alpha_{l}(s)-\Delta \alpha_{\text {had }}^{(5)}(s)\right) / \alpha(0)=128.936 \pm 0.046$. 
Table 3

Parametrization of the hadronic vacuum polarization

\begin{tabular}{cccc}
\hline range $\sqrt{s}, \mathrm{GeV}$ & $\mathrm{A}$ & $\mathrm{B}$ & $\mathrm{C}$ \\
\hline $0.0-0.7$ & 0.0 & 0.0023092 & 3.9925370 \\
$0.7-2.0$ & 0.0 & 0.0022333 & 4.2191779 \\
$2.0-4.0$ & 0.0 & 0.0024402 & 3.2496684 \\
$4.0-10.0$ & 0.0 & 0.0027340 & 2.0995092 \\
$10.0-m_{Z}$ & 0.0010485 & 0.0029431 & 1.0 \\
$m_{Z}-10000.0$ & 0.0012234 & 0.0029237 & 1.0 \\
$10000.0-100000.0$ & 0.0016894 & 0.0028984 & 1.0 \\
\hline
\end{tabular}

The most important energy range for further improvements is still the low energy region, mainly from 1 to $5 \mathrm{GeV}$ c.m.s., and efforts for more precise measurements should be strongly encouraged.

\section{Acknowledgement}

We would like to thank Zhengguo Zhao and his BES team, S.I. Eidelman from Novosibirsk and the LEP electroweak working group with Bob Clare, Martin Grünewald and Günter Quast.

\section{References}

[1] LEP Electroweak Working Group, http://lepewwg.web.cern.ch/LEPEWWG/.

[2] B. Pietrzyk, The global Fit to Electroweak Data, preprint LAPP-EXP 2000-06, to appear in the Proc. of ICHEP2000, Osaka, Japan, July 2000.

[3] M. Steinhauser, Phys. Lett. B429 (1998)158.

[4] N. Cabbibo and R. Gatto, Phys. Rev. 124 (1961) 1577.

[5] H. Burkhardt, F. Jegerlehner, G. Penso and C. Verzegnassi, Z. Phys. C43 (1989) 497.

[6] H. Burkhardt and B. Pietrzyk, Phys. Lett. B356 (1995) 398.

[7] J.Z. Bai et al., BES Collab., preprint hep-ex/0102003.

[8] J.Z. Bai et al., BES Collab., Phys. Rev. Lett. 84 (2000) 594.

[9] R.R. Akhmetshin et al., CMD-2 Collab., hep-ex/9904027. 
[10] R. Ammar et al., CLEO Collab., Phys. Rev. D57 (1998) 1350.

[11] D. Haidt, in 'Directions in High Energy Physics', Vol. 14, Precision Tests of the Standard Electroweak Model, Ed. P. Langacker, World Sc. Comp., 1995.

[12] S. Bethke - page 213, J. Casaus - page 219, D. A. Harris - page 247, Proc. of the XXXth Rencontres de Moriond, Les Arcs, March 18-25, 1995, Ed. J. Trân Thanh Van, Editions Frontieres.

[13] Review of Particle Properties, Eur. Phys. J. C15 (2000) 1.

[14] F. Jegerlehner in 'Testing the Standard Model', eds. Cvetič, Langacker, World Scientific, Singapore, 1991, p. 476.

[15] R.B. Nevzorov, A.V. Novikov and M.I.Vysotsky, JETP Lett. 60 (1994) 399.

[16] B.V. Geshkenbein and V.L. Morgunov, Phys. Lett. B340 (1994) 185, B352 (1995) 456.

[17] A.D. Martin and D. Zeppenfeld, Phys. Lett. B345 (1994) 558.

[18] M.L. Swartz, SLAC-PUB-6710, Nov. 1994, hep-ph/9411353.

[19] M.L. Swartz, Phys. Rev. D53 (1996) 5268.

[20] S. Eidelman and F. Jegerlehner, Z. Phys. C67 (1995) 585.

[21] K. Adel and F. Yndurain, FTUAM-95-32, Sept. 1995, hep-ph/9509378.

[22] R. Alemany et al., Eur. Phys. J. C2 (1998) 123.

[23] M. Davier and Höcker, Phys. Lett. B419 (1998) 419.

[24] J. Kühn and M. Steinhauser, Phys. Lett. B437 (1998) 425.

[25] S. Groote, J. G. Körner, K. Schilcher and N. F. Nasrallah, Phys. Lett. B 440 (1998) 375.

[26] M. Davier and Höcker, Phys. Lett. B435 (1998) 427.

[27] F. Jegerlehner, Hadronic Effects in $(g-2)$ and $\alpha_{Q E D}\left(M_{Z}\right)$ : Status and Perspectives, Proc. of Int. Symp. on Radiative Corrections, Barcelona, Sept. 1998, Ed. Joan Solà, World Scientific, Singapore, 1999, page 75.

[28] J. Erler, Phys. Rev. D59 (1999) 054008.

[29] A.D. Martin, J. Outhwaite and M.G. Ryskin, Phys. Lett. B492 (2000) 69.

[30] H. Burkhardt, TASSO Note 192 (1981) and DESY F35-82-03 (thesis). 


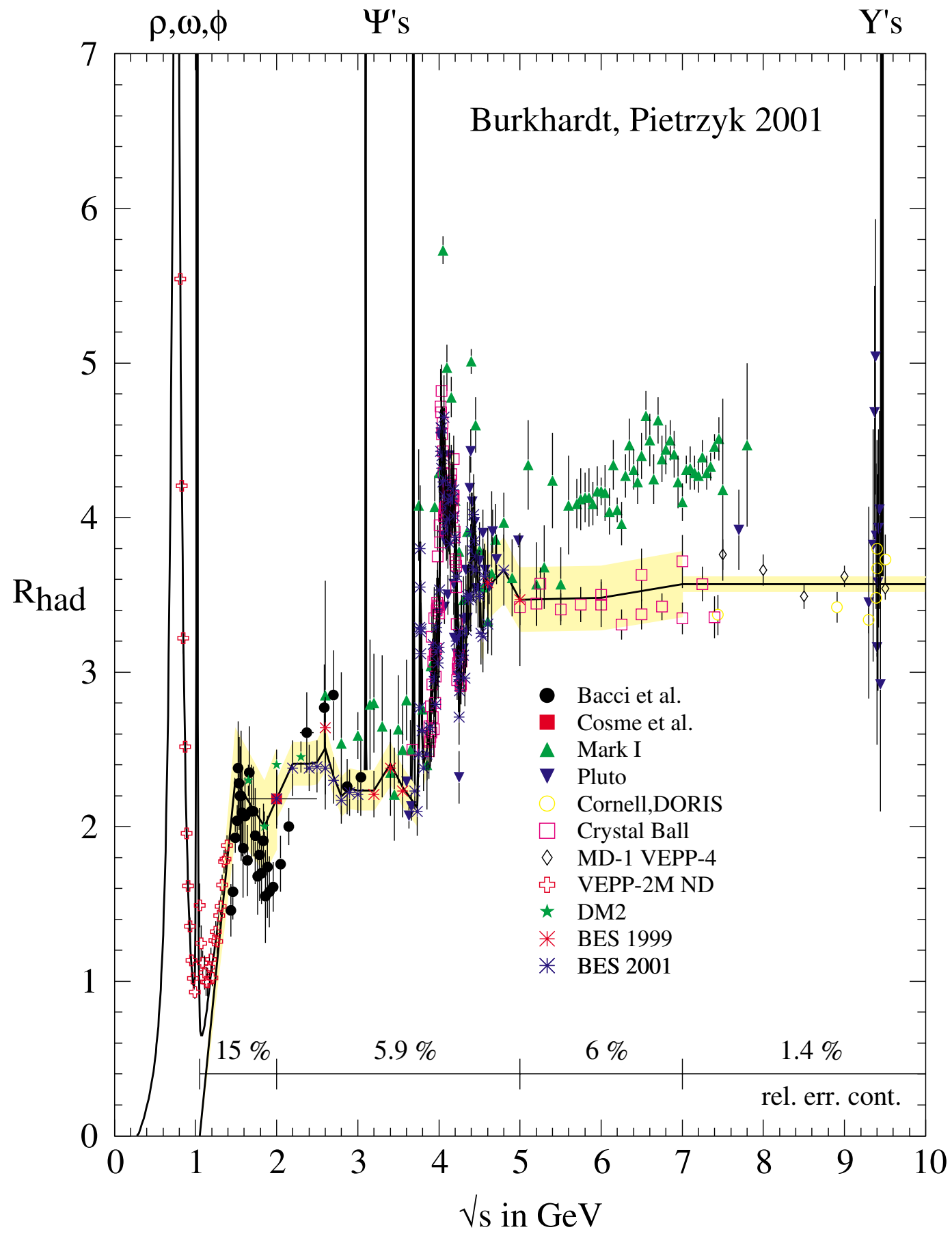

Fig. 1. $R_{\text {had }}$ including resonances. Measurements are shown with statistical errors. In addition there are overall systematic errors (up to $20 \%$ in case of Mark I). The relative uncertainty assigned to our parametrization is shown as band and given with numbers at the bottom. 
contributions at $m_{\mathrm{Z}}$

Burkhardt, Pietrzyk 2001
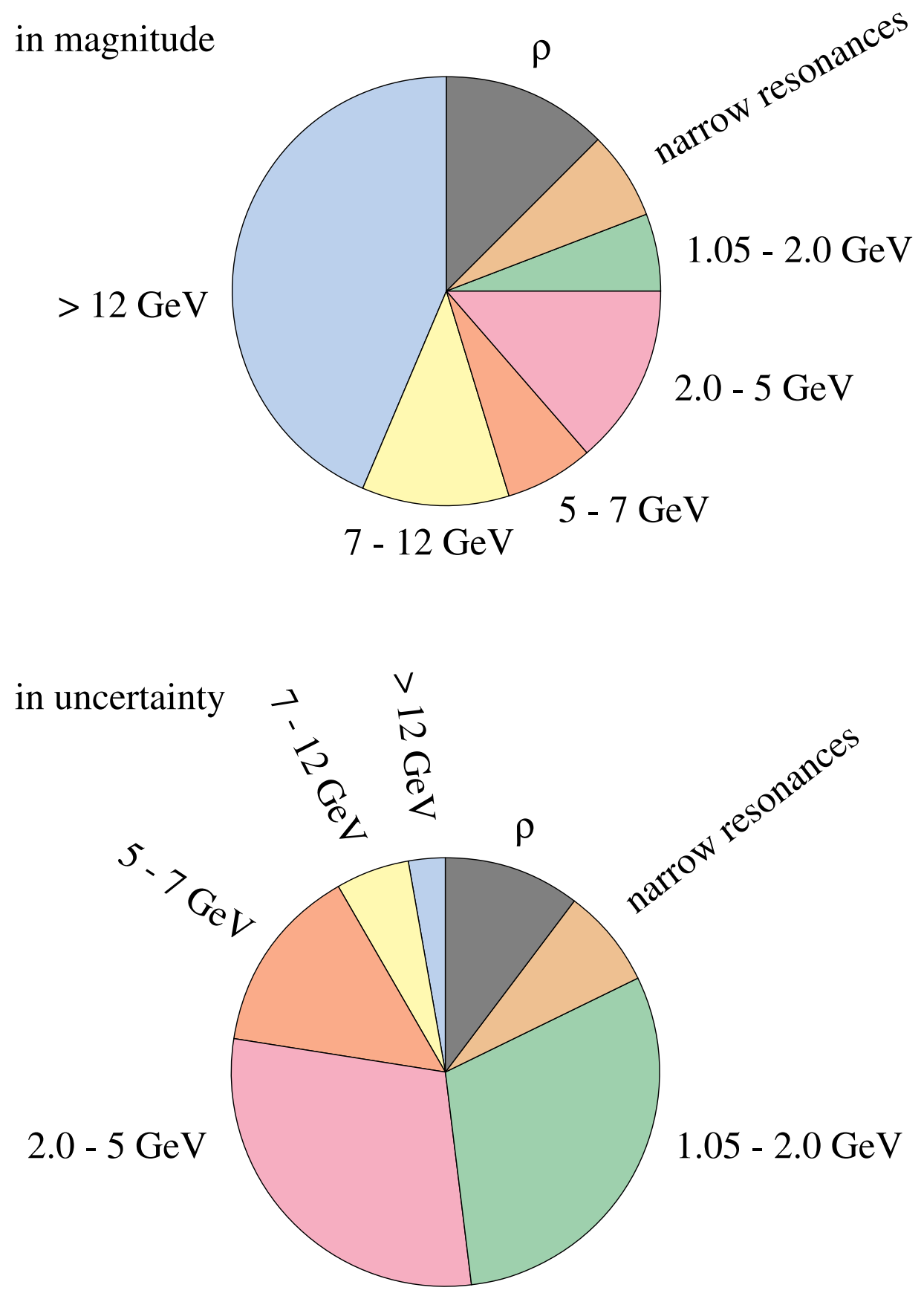

Fig. 2. Relative contributions to $\Delta \alpha_{\text {had }}^{(5)}\left(m_{Z}^{2}\right)$ in magnitude and uncertainty. 


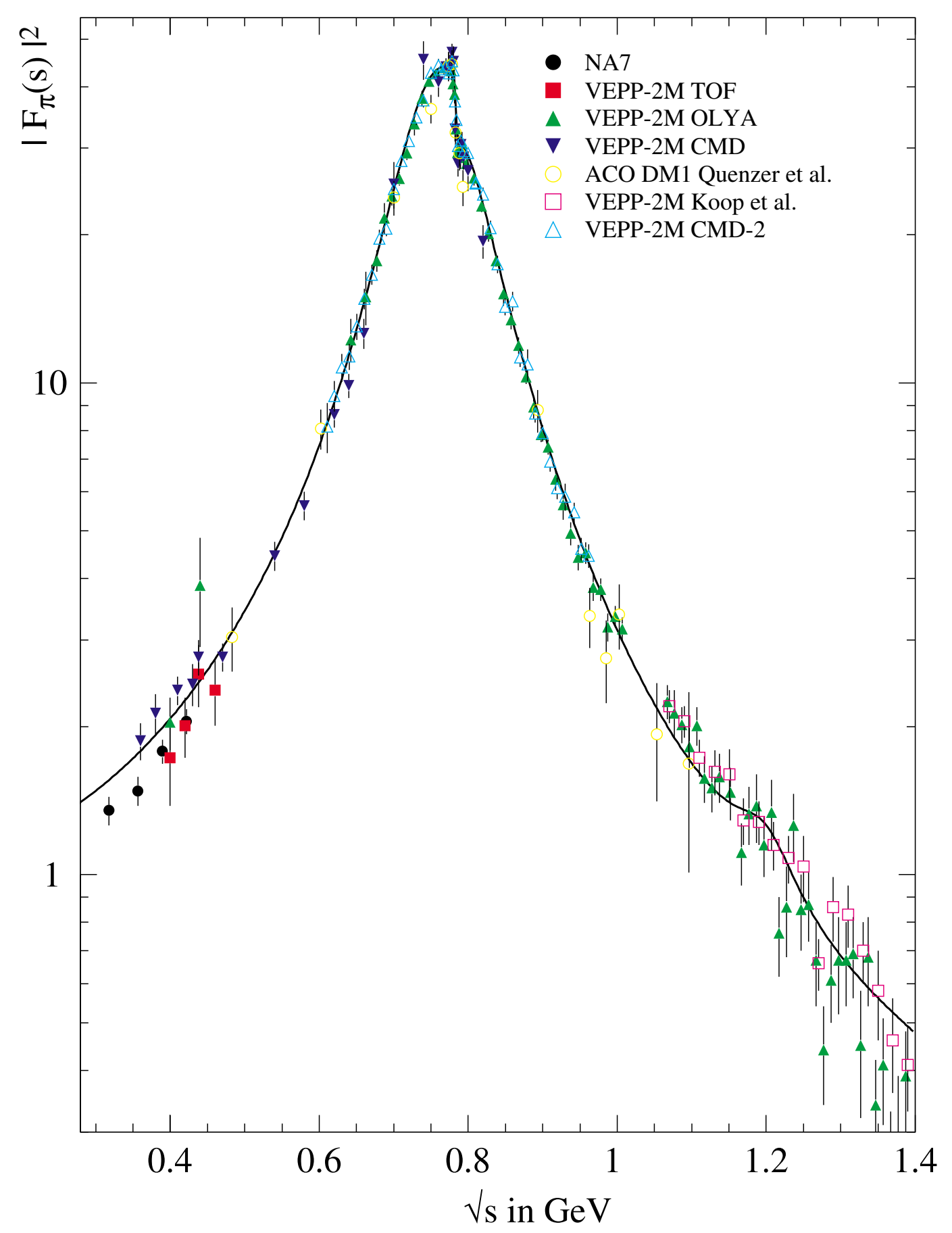

Fig. 3. The pion form factor. 


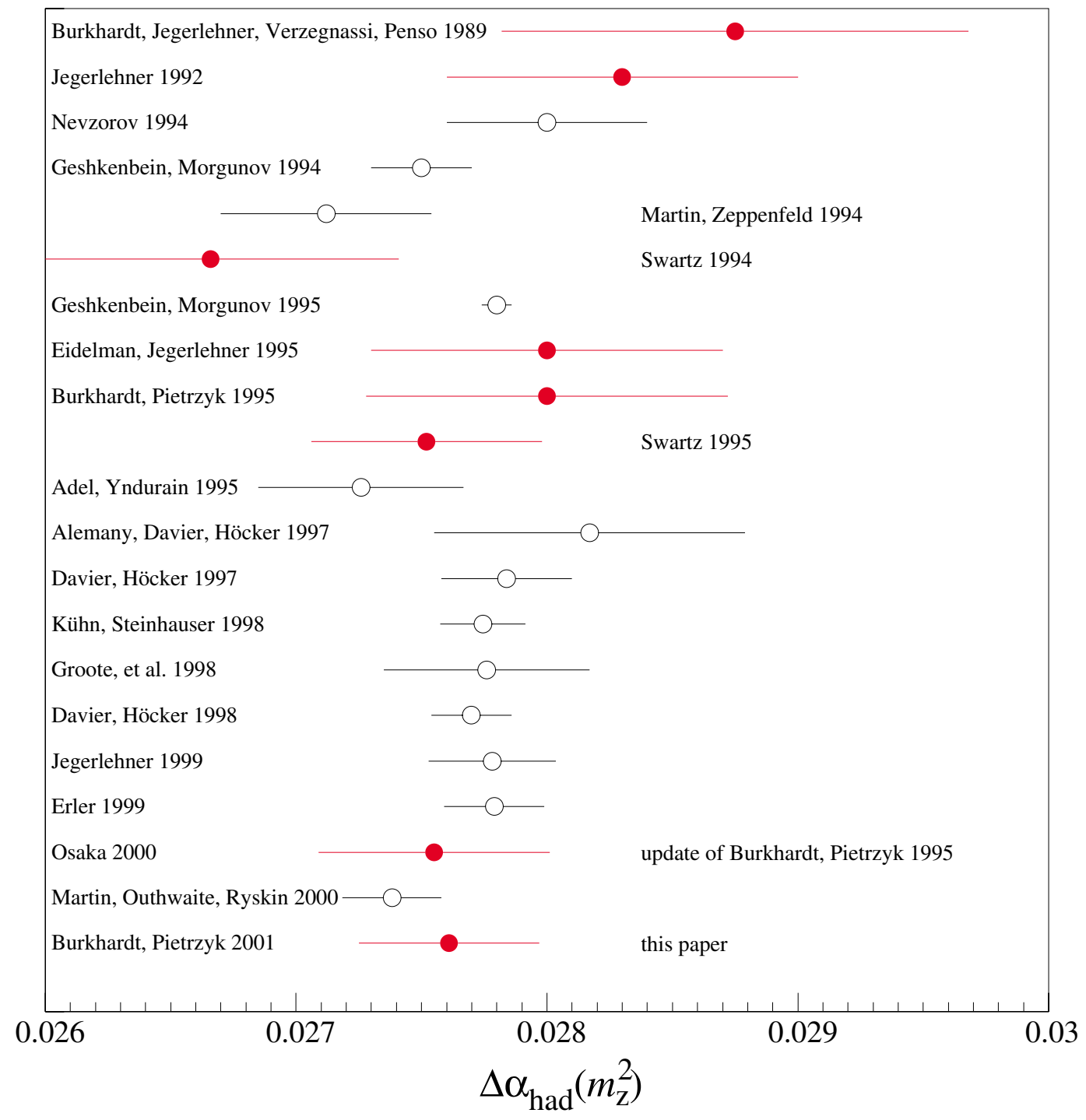

Fig. 4. Comparison of recent estimates of $\Delta \alpha_{\text {had }}^{(5)}\left(m_{Z}^{2}\right)$. Estimates based on dispersion integration of the experimental data are shown with solid dots and estimates relying on additional theoretical assumptions shown as open circles. 